\title{
Geographische Fallstudie
}

\author{
Ein neues Unterrichtsfach im Diplomstudium «Geographie» an der Abteilung für Naturwis- \\ senschaften der ETH Zürich.
}

\section{Einleitung}

Ziele des Geographiestudiums: An der Abteilung für Naturwissenschaften der ETH Zürich wurde auf Herbst 1984 das Diplomstudium in Geographie von Grund auf neu gestaltet. Die organisatorischen Gegebenheiten und die in der Tradition der Geographie stehende Ausrichtung auf den Problemkreis «Mensch und Natur» führten zur Formulierung der folgenden Ziele für das Diplomstudium: Im Grundstudium wird eine allgemeine mathematisch-naturwissenschaftliche Grundausbildung und eine Einführung in Denkweise und Methoden der Geographie vermittelt. Im allgemeinen Fachstudium wird grundlegendes Fachwissen vermittelt, werden Methoden und Techniken der Geographie gelehrt und eingeübt. Im Vertiefungsfach wird die Grundlage für eine spätere berufliche Tätigkeit oder eine weiterführende Ausbildung gelegt.

Die Einführung einer Geographischen Fallstudie im allgemeinen Fachstudium ist die eigentliche Innovation im neuen Curriculum. Das Ziel der Fallstudie ist es, daß die Studenten* allgemein umsetzbare Fähigkeiten der empirischen Forschung anhand einer aktuell vorliegenden und relevanten Fragestellung erwerben. Mit der Fallstudie sind also auf der Studienplanebene sehr allgemeine Vorstellungen verbunden, die sich etwa wie folgt umschreiben lassen: Die Studenten üben an einem «praxisorientierten Projekt», d. h. sie bearbeiten einen «Fall» als Studienobjekt, sammeln Erfahrungen in der Zusammenarbeit mit Fachleuten aus der Praxis und wenden theoretisches Wissen auf ein konkretes Problem an.

Im Herbst 1987 lagen erste Erfahrungen mit dem neuen Diplomstudium vor. Die Beurteilung war im allgemeinen positiv. Im speziellen wurde die Fallstudie von Studenten- und Dozentenseite als zwar anspruchsvolle und zeitlich aufwendige, aber im Vergleich zum übrigen Hochschulbetrieb sehr motivierende Unterrichtsform empfunden. Von den dabei gemachten Erfahrungen berichtet dieser Beitrag

\footnotetext{
* In den folgenden Ausführungen gilt, wo nicht anders angemerkt, daß mit der allgemeinen Formulierung Student/Dozent immer weibliche und männliche Personen angesprochen sind.
}

am Beispiel der ersten Fallstudie 1986/87. Vereinzelt werden auch Erfahrungen aus der zweiten Fallstudie 1987/88 zur Illustration einiger Punkte herangezogen.

Beide Fallstudien wurden von E. Buff Keller und P. Pfister als verantwortliche Dozenten geleitet; H. Gilgen war maßgebend an der Erstellung des Konzeptes für diese Innovation im Geographiestudium an der ETHZ beteiligt.

\section{Pädagogische und didaktische Zielvorstellungen}

Mit der Einführung des Faches «Geographische Fallstudie» werden die zwei folgenden Leitideen verfolgt: Zum einen soll im Sinne des forschenden Lernens ein kleines, praxisorientiertes Forschungsprojekt durchgeführt werden. Diese Lehrveranstaltung ist also nicht als «Vorlesung über Fallstudien» konzipiert, sondern es soll konkret eine Fallstudie durchgeführt werden. Zugleich sollen damit bereits im Studium soziale Fähigkeiten wie Kooperation, interdisziplinäre Zusammenarbeit, Konfliktlösungskompetenzen usw., geschult werden. Diese Fähigkeiten sind wichtig für eine spätere Berufstätigkeit. Sie werden mit Vorteil im Gruppenunterricht geschult. Zum anderen soll die Wirkung von verschiedenen Unterrichtsformen, im speziellen des Team-Teachings und des arbeitsteiligen Gruppenunterrichts, empirisch erforscht werden.

Team-Teaching: Die geforderte Praxisrelevanz eines solchen Unterrichts-Projekts impliziert, daß es von zwei Dozenten im Team-Teaching geleitet wird. Der erste Dozent soll ein Geograph mit didaktischer und pädagogischer Ausbildung sein, welcher die Fallstudie seitens des Geographischen Instituts betreut. Der zweite Dozent soll ein in der Praxis stehender Fachmann mit Fähigkeiten zu interdisziplinärer Zusammenarbeit sein. Er soll Projektleiter in einem Planungsbüro oder einem öffentlichen Amt

Eva Buff Keller, Dr., und H. Gilgen, Dr., Geographisches Institut ETH. Winterthurerstr. 190, 8057 Zürich; P. Pfister, Arcoplan, Zürcherstr. 25, 5400 Baden 
sein und über ein geeignetes Projekt verfügen.

Eine Lehrveranstaltung im Team-Teaching zu leiten, bietet viel Anregung und Lernchancen, beinhaltet aber auch Bedrohungen und emotionale Gefahren für die Lehrenden. DIEM-WILLE (1986: 177) beschreibt die vielen Seiten des Team-Teachings in eindrücklicher Weise: "Eine interdisziplinäre Lehrveranstaltung zu leiten, heißt für beide Wissenschafter, an die Grenzen ihrer Disziplin zu kommen, wo sie meist keine Experten mehr sind. Die gleichzeitige Anwesenheit eines zweiten Lehrenden kann als Infragestellung der eigenen Autorität erlebt werden. Auf alle Fälle wird der eigene hochschuldidaktische Ansatz sichtbar; die Art und Weise, mit Studenten umzugehen wird befragbar und durch den zweiten Lehrer relativiert. Wer kommt bei den Studenten besser an? Gibt man sich vor dem Kollegen eine Blöße, wenn man etwas nicht weiß? Kann man die Bedeutung des eigenen Fachs bei der interdisziplinären Fragestellung sichtbar machen? Die angeführten emotionalen Belastungen stellen eben dadurch, daß man dabei auf die Grenzen seines Wissens oder seiner didaktischen Fähigkeiten stößt, auch Lernchancen dar. Man lernt eine andere Gestaltung der Lehrveranstaltung kennen, ist gezwungen, Fragen neu zu überdenken, kann mit dem anderen seine Eindrücke über die Lehrveranstaltung und die auftretenden Probleme besprechen.»

Unterrichtsformen: Vorlesungen der leitenden Dozenten im Frontalunterricht werden in jedem Semester durch ein bis zwei Gastreferate ergänzt. Die eingeladenen Referenten gewähren an problemorientierten Beispielen den Studenten Einblick in ihre Tätigkeit. Die wichtigste Unterrichtsform bildet die arbeitsteilige Gruppenarbeit, auf die weiter unten noch ausführlicher eingegangen wird. Zusätzlich werden auch Exkursionen durchgeführt, bei denen einerseits der Kontakt mit in der Praxis stehenden Fachleuten und andererseits das Studium der Untersuchungsobjekte vor Ort im Mittelpunkt stehen. Einen weiteren Höhepunkt - und bezüglich der Motivation wichtige Unterrichtsform - bildet die Durchführung einer Feldwoche, auf die ebenfalls noch näher eingegangen wird. Nach der Feldwoche, die in erster Linie der Datenerhebung und Durchführung der eigentlichen Untersuchung im Forschungsgebiet dient, werden die erhobenen Daten ausgewertet und die Ergebnisse in Form eines wissenschaftlichen Berichtes dargestellt. Dieser muß den ganzen Aufbau und Ablauf des bearbeiteten Projekts enthalten. Die Fallstudie findet ihren Abschluß in einer Präsentation der Studie und ihrer Ergebnisse.

Gruppenunterricht: Im heutigen geographischen Verständnis steht der Mensch als gesellschaftlich raumwirksames Wesen im Zentrum. Deshalb soll im Fach Geographie ein handlungsorientierter und so- mit studentenzentrierter Unterricht Priorität haben. Leider wird beim großen Anteil der Vorlesungen in den Studienplänen der ETH die Selbsttätigkeit der Studenten zuwenig gefördert, mit dem Ergebnis, daß wissenschaftliche und soziale Fähigkeiten zu wenig geübt werden. Ein Gegengewicht soll mit dem arbeitsteiligen Gruppenunterricht in der Fallstudie gesetzt werden. Mit dieser Unterrichtsform läßt sich die Fähigkeit zur Problemdifferenzierung sehr gut schulen: Es können mehrere Lösungsvorschläge und Alternativen entwickelt werden. Dadurch wird die Kreativität angeregt. Das Feedback in der Gruppe führt meist auch zu einer Steigerung der Leistung des einzelnen (LEMERHOFER 1986: 195). Weiter wird die Fähigkeit geübt, Kritik entgegenzunehmen und andere konstruktiv zu kritisieren. Diese Möglichkeiten des Gruppenunterrichts fördern die Fähigkeit zur Kooperation, die auch nachher in der Praxis von großer Bedeutung sein wird (BUFF KELLER 1987: 11). Durch die Unterstützung des Wunsches nach Selbstbestimmung wird schließlich das Ziel erreicht, daß die Studenten die Verantwortung für das Projekt schrittweise selbst übernehmen.

Im interdisziplinär im Team-Teaching geführten Gruppenunterricht geben die Dozenten die sicherheitsspendende Reglementierung der Vorlesung auf. Damit tritt die soziale Dimension der Lehrveranstaltung in den Vordergrund, die Sensibilität und Fähigkeiten im Umgang mit gruppendynamischen Prozessen erfordert. Damit ist auch ein gewisses Maß des «Nicht-planen-Könnens» und der Unsicherheit auszuhalten, was Ich-Stärke und Angstfreiheit erfordert. Im weiteren kann eine unbewußte Angst hinzukommen, überflüssig zu sein und sein Wissen nicht zeigen zu können. Aus diesem Grund bedarf es noch größerer Kompetenz, um die Studenten zu selbstbestimmtem Lernen zu führen (DIEM-WILLE 1987: 184).

Ein erster Schritt zu selbstbestimmtem Lernen ist bereits die Bildung der Gruppen. Die Studenten wählen sich gegenseitig nach Sympathie und/oder Forschungsinteressen aus, um eine ebenfalls selbst gewählte Forschungsfrage zu bearbeiten. Je mehr die einzelnen Vorstellungen der Studenten in die Gruppenarbeit einbezogen werden können, um so größer ist die Identifikation mit dem Projekt. Entsprechend stärker ist dann auch das Durchhaltevermögen gegenüber auftretenden Schwierigkeiten. Im Sinne eines forschenden Lernens ist der Prozeß der Entscheidungsfindung für die relevante Forschungsfrage, die Bildung der Gruppe, das Formulieren der Vorgehensweise und der Prozeß der Forschung ebenso wichtig wie das letztlich erreichte und in den Berichten dargestellte Resultat.

In Kap. 5 wird über die Erfahrungen berichtet, die während der Fallstudie 1986/87 mit den verschiedenen Unterrichtsformen gemacht wurden. 


\section{Inhaltliche und methodische Ziele}

Bei der Definition der inhaltlichen und methodischen Ziele, die für die Fallstudie 1986/87 und die Themenwahl maßgebend sein sollten, gingen wir von folgenden Prämissen aus: der in Kap. 1 vorgestellten Definition der Fallstudie und den damit verknüpften allgemeinen Vorstellungen, den durch den Studienplan gegebenen Rahmenbedingungen, sowie den in Kap. 2 enthaltenen pädagogischen und didaktischen Vorgaben. Als weitere wichtige Vorgabe war einzuhalten, daß die Studenten in ihrer Situation an einer Hochschule möglichst viel von den Randbedingungen erfahren sollten, welche für Forschungsaufgaben in der Praxis gelten. Die derart gefundenen Ziele werden im folgenden im Überblick dargestellt. Sie erhielten unterschiedliche Bedeutung, je nach dem Thema, das von einer Gruppe gewählt wurde.

Praxisrelevanz: Die Forschungsaufgabe soll eine aktuelle Fragestellung oder einen Teilbereich davon betreffen, die wenn möglich Gegenstand eines laufenden oder vorgesehenen Projekts ist. Sie soll ein Beitrag zur praktischen Umweltforschung sein.

Standort und Verhalten des Wissenschaftlers: Das Thema muß sich eignen, die Rolle und die Kompetenz des Wissenschaftlers und dessen Grenzen an einem konkreten Beispiel aufzuzeigen.

Adressaten, Kontaktpersonen, Feedback: Die Forschungsaufgaben sollen in bestehende institutionelle Strukturen eingebettet werden.

Problemdefinition: Die Aufgabe soll nicht vordefiniert, sondern erst im Ansatz oder in Form einer generellen Fragestellung eingebracht werden. Die Formulierung und die Definition der Forschungsaufgabe sollen Teil des gesamten Forschungsablaufes sein.

Komplexitätsanforderung: Die Fallstudie soll ein Thema aufgreifen, das nur auf der Basis des Vernetzungsaspektes der Probleme erfolgreich behandelt werden kann. Dabei ist darauf zu achten, daß das Thema auf die vorhandenen Kapazitäten und Möglichkeiten einschränkbar ist.

Effizienzproblem: Die in der Praxis bestehenden finanziellen und weiteren Beschränkungen sollen durch die Zeitlimite und den begrenzten Personeneinsatz simuliert werden.

Kreativitätsanforderung: Trotz anfangs fehlender Grundlagen (Unterlagen, Daten, Kontaktmöglichkeiten usw.) sollen konkrete Aussagen oder Schritte gemacht werden, die in der Fragestellung weiterhelfen.

Prozeßorientierung: Für die Bewertung der Arbeiten sind nicht nur die Resultate in Form von Berichten, Plänen usw. wichtig, sondern auch der Prozeß des forschenden Lernens. Das methodische Vorgehen bei der Bearbeitung des Themas, die Interaktionen und die Konfliktbewältigung in der Gruppe sind von gleicher Bedeutung wie die Resultate.

Mit diesen inhaltlichen und methodischen Zielen war die Fallstudie 1986/1987 im Ansatz vorgegeben und der Begriff «praxisorientiert» für die Themenwahl genügend präzisiert.

\section{Themen der Fallstudie 1986/87}

Allgemeines: Unter dem Aktualitäts- und dem Vernetzungsaspekt bot sich als übergeordnetes Thema der Bereich «Gewässer» an. Sowohl in seiner Bedeutung für die gegenwärtige Umweltpolitik als auch in seinen gesamträumlichen Zusammenhängen weist dieses Thema auf wichtige künftige Forschungsaufgaben hin, die mit Ausnahme verschiedener Sachfragen erst in Konturen sichtbar werden. $\mathrm{Daß}$ durch diese Fragen im Bereich «Gewässer» auch den institutionellen Gremien (öffentliche Verwaltung usw.) dauernd neue Aufgaben gestellt und die Grenzen institutionellen Handelns erkannt werden, war ein weiterer Ausgangspunkt für die Themenwahl. So wird z. B. im Kt. Thurgau darüber diskutiert, welche Aspekte in einer künftigen Gewässerschutzpolitik wichtig sind, welche Zusammenhänge bestehen und welche Voraussetzungen an den Gewässerschutz gestellt werden müssen.

Bei der Diskussion dieses übergeordneten Themas bildeten sich drei Gruppen. Jede der Gruppen wählte aus der weitreichenden Problematik ein Thema unter der Bedingung, daß nicht zwei Gruppen dasselbe wählten. Damit wurde erreicht, daß in den Gruppenarbeiten unterschiedliche Forschungsinteressen berücksichtigt und durch die Auswahl eine Erweiterung des Erfahrungshorizonts erreicht werden konnte. Als Themen wurden vorgeschlagen:

- Gewässernetz. Entwicklung, Bewertung. Schwergewicht: geographisch/ökologische Bedeutung am Beispiel Seebachtal.

- Grundwasser. Nutzung, Veränderung. Schwergewicht: hydrologische Analyse der Problemsituation im Thunbachtal.

- Gewässerumwelt. Zusammenhänge, Bedrohungen der Gewässer. Schwergewicht: Analyse der Einflußfaktoren.

Der Einstieg in das allgemeine Thema «Gewässer» erfolgte mit der Methode des vernetzten Denkens. Dieser Ansatz wurde auch in der folgenden Untersuchung und Bearbeitung der Thematik angewendet. Im Anschluß daran mußten methodische Ansätze gefunden werden, um aus der komplexen Ausgangssituation Sachfragen abzuleiten, die als Beitrag zur aktuellen Gewässerpolitik in den untersuchten Gebieten gelten konnten. Das Resultat der 
Gruppenarbeiten ist in der Geographischen Fallstudie BUfF KELLER und PFISTER (Hrsg., 1988) enthalten. Im folgenden sind Zusammenfassungen der Berichte dargestellt.

Entwicklung und Bewertung des Gewässernetzes im Seebachtal: Als Untersuchungsgebiet wurde das rund $30 \mathrm{~km}^{2}$ umfassende Einzugsgebiet des Seebachs im Kanton Thurgau gewählt. Dieses Gebiet ist im Bundesinventar der Landschaften von nationaler Bedeutung enthalten. Der Eintrag im Inventar schützt jedoch die Gewässer nur zum Teil vor Veränderungen, wie weiter unten dargelegt wird. In weiter zurückliegender Zeit, d. h. vor dem Eintrag, war gar kein Schutz vorhanden. Aus diesem Grund wurde anhand von Siegfriedkarten, Landeskarten und Luftbildern der Landestopographie die quantitative Entwicklung des Gewässernetzes seit 1900 untersucht (Tab. 1). Infolge der intensiveren Landnutzung, Besiedlung und Kulturlandgewinnung wurden sehr viele Bachläufe eingedolt oder verschwanden durch künstliche Entwässerung völlig. Bei Feldbegehungen wurden über $10 \%$ (bezogen auf die Gesamtlänge der Fließgewässer) zusätzliche
Bachläufe gefunden, d. h. Gewässer, die nicht auf der Landeskarte eingetragen sind. Diese meist kleinen Bäche sind bei Meliorationen besonders gefährdet, da sie nicht nachgewiesen werden können. Man

Tab. 1 Absolute und prozentuale Abnahme der Länge aller Fließgewässer. Die Zahlen beziehen sich auf sämtliche oberirdische Fließgewässer im Einzugsgebiet des Seebachs. Die Länge aller Fließgewässer um 1904 wurde als 100\% angenommen.

\begin{tabular}{|c|c|c|c|c|c|}
\hline Jahr & $1891 / 1904$ & \multicolumn{2}{|c|}{1945} & $1957 / 58$ & 1984 \\
\hline offene Fliessgewăsser & $50,2 \mathrm{~km}$ & \multicolumn{2}{|c|}{$39,6 \mathrm{~km}$} & $24,4 \mathrm{~km}$ & $18,7 \mathrm{~km}$ \\
\hline Abnahme absolut & \multicolumn{2}{|c|}{$10,6 \mathrm{~km}$} & \multicolumn{2}{|c|}{$15,2 \mathrm{~km}$} & $5,7 \mathrm{~km}$ \\
\hline $\begin{array}{l}\text { Abnahme in \%, wobei } 100 \% \text { die Långe } \\
\text { der Fliessgewässer } 1891 / 1904 \text { ist }\end{array}$ & \multicolumn{2}{|c|}{$21,1 \%$} & \multicolumn{2}{|c|}{$30,2 \%$} & $11,3 \%$ \\
\hline \multicolumn{3}{|l|}{ Abnahme absolut $1891 / 1904$ bis 1984} & \multicolumn{3}{|c|}{$31,5 \mathrm{~km}$} \\
\hline Abnahme in \% 1891/1904 bis 1984 & \multicolumn{5}{|c|}{$62,6 \%$} \\
\hline
\end{tabular}

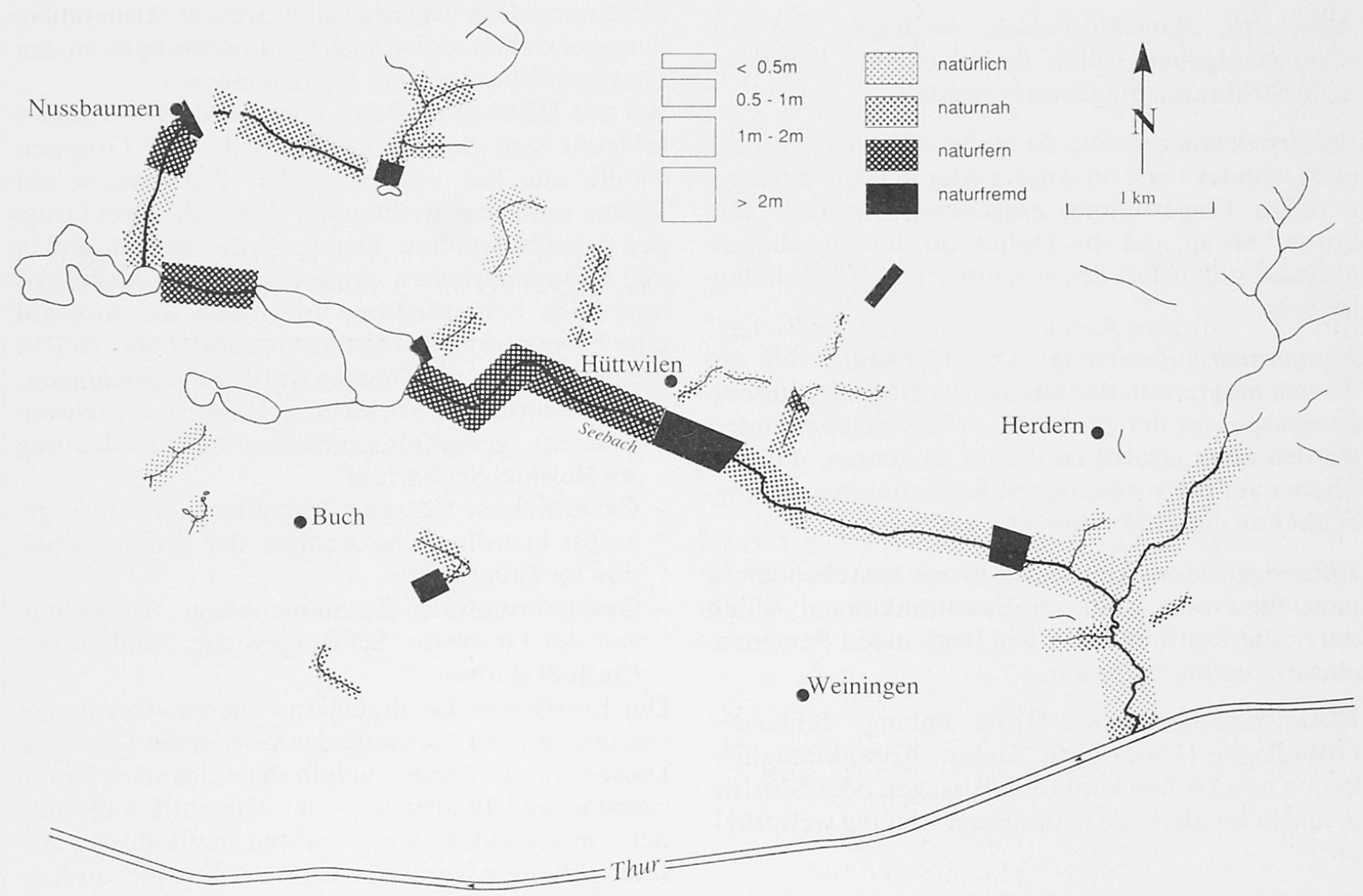

Abb.1 Resultate der Bewertung der Fließgewässer im Seebachtal (überarbeitet nach Buff Keller und Pfister 1988: 24a) 
stellte aber auch Veränderungen der Art und des Aussehens der Bäche fest. Deshalb wurde ein Bewertungsverfahren entwickelt, um damit die Qualität der Bachläufe festzuhalten. Jedes in der Qualität konstante Stück eines Bachlaufes wird auf einem Inventarblatt anhand der Kriterien Sohlenart, Profil, Linienführung, Verbauungsgrad und Bewuchs der Ufer einer der vier Kategorien «natürlich», «naturnah», «naturfern» oder «naturfremd» zugeordnet.

Die Resultate dieser Bewertung wurden in einer Karte (Abb.1) dargestellt, welche zusammen mit den Inventarblättern als Grundlage für künftige Planungs- und Schutzmaßnahmen dienen kann. Ebenso ist es möglich, die Entwicklung weiter zu verfolgen, da nun eine Art Referenzzustand beschrieben ist.

Grundwasserneubildung im Thunbachtal: Im Thunbachtal ist das Problem der Grundwasserknappheit schon lange bekannt. Schon die beiden ersten Grundwasserfassungen 1925 und 1926 führten zu einer drastischen Absenkung des Grundwasserspiegels. Markante Drosselungen der Fördermengen von anfangs $4000 \mathrm{l} / \mathrm{min}$ auf heute 200 bis $250 \mathrm{l} /$ min ergaben nicht den gewünschten Effekt. Aufgrund der akuten Lage erhielt das Geologie-Büro Büchi \& Müller in Frauenfeld den Auftrag, die Situation zu untersuchen. Sämtliche bisher gesammelten Daten standen dieser Gruppe zur Verfügung, um die Veränderung des Grundwasserspeichers mit Hilfe der hydrologischen Grundgleichung abzuschätzen. Dazu wurde die hydrologische Grundgleichung mit anthropogen bedingten Parametern ergänzt:

$$
\mathrm{P}+\mathrm{T}=\mathrm{Ro}+\mathrm{Ru}+\mathrm{K}+\mathrm{ET} \pm \mathrm{S}
$$

P Niederschlag

Ro oberirdischer Abfluß

T Trinkwasserzufuhr

$\mathrm{Ru}$ unterirdischer Abfluß

K Abwasser, zur Kläranlage ET Evapotranspiration

\section{S Speicheränderung}

Der wichtigste Parameter, aber zugleich auch der unsicherste, ist die Evapotranspiration. Sie kann nicht direkt gemessen werden, wie etwa der Abfluß oder der Niederschlag, sondern muß mit einer Formel näherungsweise berechnet werden. Die Berechnung erfolgte mit den drei Formeln nach Haude, Schendel und Turc (HÖLTING 1984: 28ff.), mit Daten, die an den Stationen der Schweizerischen Meteorologischen Anstalt in Tänikon (Monatsmittel der Lufttemperatur und der Lufttemperatur um 13 Uhr), in Tänikon und Güttingen gemittelt (Monatsmittel der relativen Feuchte und der Feuchte um 13 Uhr) und Thundorf (Monatssummen des Niederschlags) gemessen wurden. Die Resultate sind in Tab. 2 dargestellt.
Zusätzlich zu diesen Berechnungen wurde ein eigenes Verfahren entwickelt, um die Verdunstung zu berechnen: die Flächennutzungsmethode. Hier wird das Untersuchungsgebiet in verschiedene Landnutzungskategorien eingeteilt, von jeder Kategorie ist die spezifische Verdunstung bekannt. Die Gesamtverdunstung ergibt sich dann durch die Summe der Flächen der Landnutzungskategorien, gewichtet mit den jeweiligen spezifischen Verdunstungen. Man erstellte mit Luftbildern und Feldarbeit eine Karte mit einer Einteilung des Untersuchungsgebietes in die vier Kategorien «Siedlung», «Wald», «Acker» und «Wiese», mit den spezifischen Verdunstungen 0, 600, 593 und 774 mm/Jahr. Durch Planimetrieren und Rechnen erhielt man das Resultat, das ebenfalls in Tab. 2, Kolonne 4, dargestellt ist.

Die Resultate aller Berechnungen liegen nahe beieinander und ergeben eine eher negative Speicheränderung (Tab.2). Die Speicheränderung im Jahr $1985 / 86$ ist etwa von derselben Größenordnung wie die geförderten Trinkwassermengen (1983/84: 17.5, 1984/85: 21.2, 1985/86: wegen Grundwasserpumpversuchen nur $3 \mathrm{~mm} / \mathrm{Jahr}$ ). Daraus kann man vorläufig schließen, daß die Förderung der Grundwasserfassungen einen direkten Einfluß auf den Grundwasserspiegel hat. Weitere, bestimmtere Aussagen sind bei einer Vergrößerung der Datenbasis möglich, was eine Weiterführung der Untersuchungen um drei bis fünf Jahre bedingen würde.

Tab. 2 Resultate der Evapotranspirationsberechnung und der Speicheränderung des Grundwassers.

\begin{tabular}{|c|c|c|c|c|}
\hline Evapotranspiration & Haude & Schendel & Turc & $\begin{array}{l}\text { Flachennutzungs } \\
\text { methode }\end{array}$ \\
\hline Oktober [mm/Mt] & 24 & 47 & - & - \\
\hline November & 9 & 4 & - & \\
\hline Dezember & 13 & 15 & - & \\
\hline Januar & 10 & 1 & - & \\
\hline Februar & 5 & -34 & - & - \\
\hline März & 26 & 16 & - & - \\
\hline April & 28 & 36 & & - \\
\hline Mai & 74 & 88 & - & - \\
\hline Juni & 78 & 104 & - & - \\
\hline Juli & 89 & 114 & & - \\
\hline August & 77 & 109 & - & \\
\hline September & 53 & 80 & - & - \\
\hline Jahr [mm/J] & 492 & 583 & 469 & 620 \\
\hline Speicheranderung & -55 & +33 & .77 & +73 \\
\hline
\end{tabular}


Bodenversiegelung in Weiningen: Es wurde die Beziehung zwischen Siedlungen und Gewässern, speziell der Einfluß der Bodenversiegelung auf den Abfluß, am Beispiel der Gemeinde Weiningen im Kt. Thurgau untersucht. Den ersten Schritt der Datenerfassung bildete eine einwöchige Feldbegehung. Es wurde die Bodenbedeckung auf Plänen im Maßstab 1:2000 und 1:5000 nach den Kategorien «Gebäude», «Straßen, Vor- und Parkplätze asphaltiert», «Schotterstraßen», «Hofgärten, Rasen, Umschwung», «Äcker, Wiesen, Weiden», «Reben», «Wälder, Feldgehölze» und «Moore, Sümpfe, Naturschutzzonen» kartiert (Abb. 2). Um die Veränderung der Bodenbedeckung festzuhalten, wurde dieselbe Kartierung (mit zusammengefaßten Kategorien) anhand von Luftbildern aus den Jahren 1935 und 1978 durchgeführt. Dabei wurde der Fehler, der durch die Luftbildgeometrie bedingt ist, vernachlässigbar klein gehalten, indem die Luftbilder mittels eines Planvariographen auf eine Tochterpause des Situationsplanes 1:5000 projiziert und nur genü-

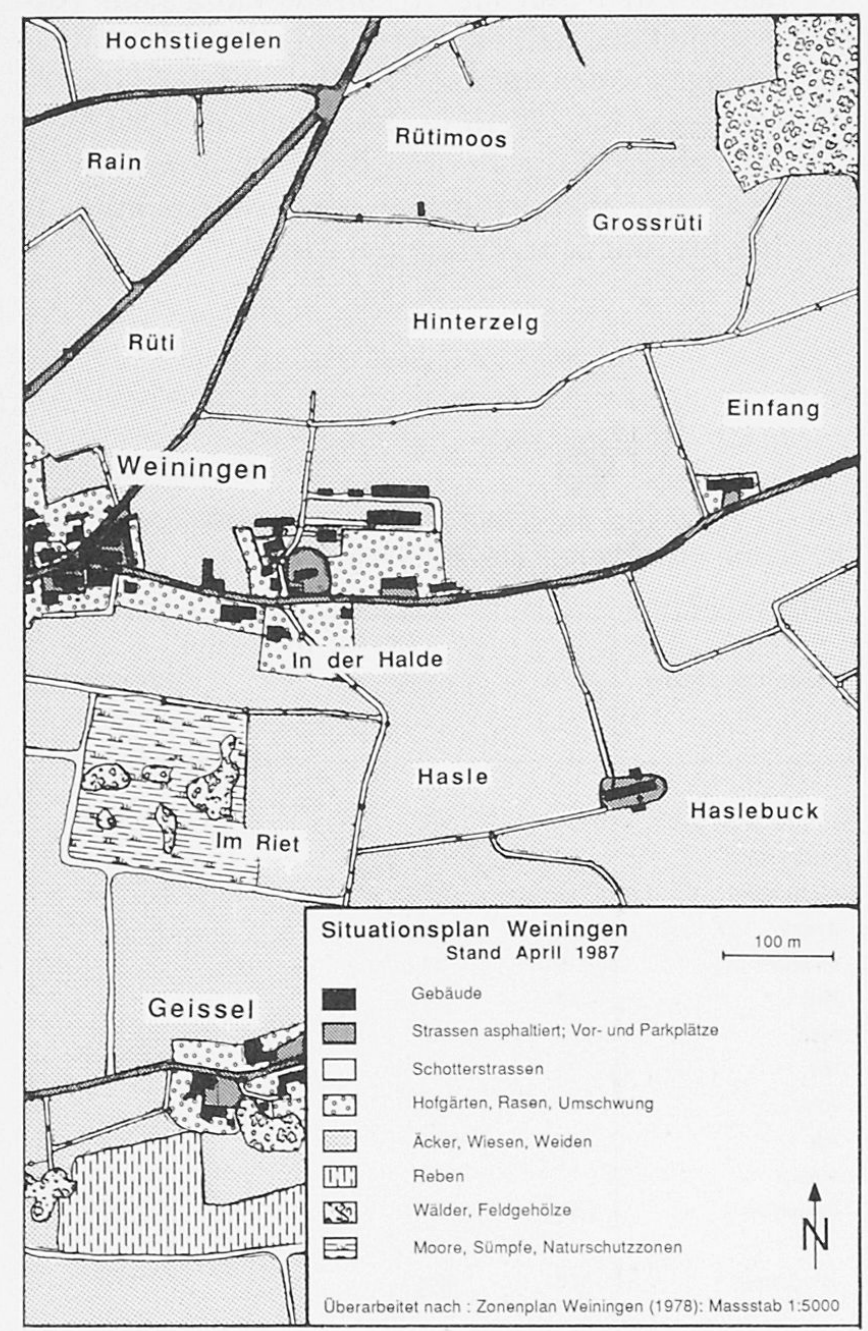

Abb. 2 Bodenbedeckung der Gemeinde Weiningen (überarbeitet nach Buff Keller und Pfister, 1988: 88) gend kleine Gebiete, laufend an den Projektionsmaßstab angepaßt, bearbeitet wurden.

Betrachtet man die Veränderung der Bodenbedekkung in Weiningen zwischen 1935 und 1978, so fällt die starke Zunahme der «überbauten Fläche» (faßt die obigen Kategorien «Gebäude», «Straßen, Vorund Parkplätze asphaltiert» und «Hofgärten, Rasen, Umschwung») von 6 auf 15 ha auf, was einer Zunahme von $150 \%$ entspricht. Ein allfälliger $\mathrm{Zu}-$ sammenhang dieser Zunahme mit Veränderungen in den Abflußkurven konnte aus Zeitmangel nicht mehr untersucht werden.

\section{Pädagogische und didaktische Erfahrungen}

In diesem Kapitel wird von den Erfahrungen berichtet, die wir mit unserem didaktischen Ansatz in der Interaktion mit den Studentinnen und Studenten während der ersten und teilweise auch der zweiten Fallstudie gemacht haben. Es werden die in Kap. 2 und 3 erwähnten Unterrichtsformen und Zielanforderungen betrachtet.

Vorlesungen: In den ersten Wochen der Fallstudie standen Vorlesungen im Stil des Frontalunterrichts sowie zwei Exkursionen und ein Gastreferat im Vordergrund. Diese Unterrichtsformen dienten in erster Linie der Informations- und Wissensvermittlung. Sie führten in das übergeordnete Thema «Gewässer» ein.

Gruppenarbeit: Als in der folgenden Phase einzelne Forschungsfragen zum Thema «Gewässer» definiert werden sollten, zeigten die Studenten unterschiedliche Verhaltensweisen. Wie bereits in Kap. 2 erwähnt, bildeten sie die Gruppen nach Sympathie und Interesse an den einzelnen Teilfragen. Diese Aufgabe, im Gruppenprozeß ihren eigenen Forschungsschwerpunkt zu definieren, war für sie neu und mit Unsicherheiten befrachtet, wobei diese Gefühle - je nach Student und Gruppe - unterschiedliche Reaktionen auslösten.

Es gab Studenten, welche sich mit der absichtlich wenig vorgegebenen Fragestellung auseinandersetzten. Sie suchten über «Versuch und Irrtum» nach einer sinnvollen Forschungsfrage, formulierten ihren Forschungsplan in Diskussionen mit der Dozentin und dem Dozenten und übernahmen die Verantwortung für ihre Arbeit. Andere Studenten begegneten in dieser eher kreativen Phase, in welcher das in den Vorlesungen geübte Verhaltensmuster nicht genügte, eigenen Unzulänglichkeiten. Sie fühlten sich überfordert oder sahen einen riesigen Berg vor sich und fanden keinen Anfang. Wieder andere überschätzten ihre Möglichkeiten, was auch dazu führen konnte, daß es nur schwer gelang, zu einer klaren Forschungsfrage vorzustoßen. 
Allgemein stellten wir fest, daß die Studenten das in den bisher besuchten Vorlesungen erworbene Wissen zu wenig auf spezielle Problemstellungen anwenden konnten. Sie konnten also vieles, was sie bereits gehört hatten, nicht in das praktische Beispiel umsetzen. Die allgemeine Entmutigung wurde z. T. verstärkt durch die Ungewißheit der zu erwartenden Resultate oder auch durch Unsicherheiten über Kritik und Beurteilung, da die Fallstudie ein Prüfungsfach ist. Auch die Angst vor Unwissenschaftlichkeit, die u. a. durch eine andere Methodenwahl oder verschiedene wissenschaftstheoretische Standpunkte hervorgerufen wurde, wirkte bei einigen Studenten als Verunsicherung.

Alle diese neuen Situationen hatten, je nach Student, verschiedene Folgen wie etwa Denkblockaden, ineffizientes und unmethodisches Vorgehen, Ausweichen vor der eigenen Unzulänglichkeit, Angst, die Verantwortung für sich und die eigene Gruppe zu übernehmen und die Neigung, nach einem andern als Schuldigen zu suchen. Als ein weiteres Problem erwies sich die Einstellung zu Fehlern. Das Lernen an unseren Gymnasien und Hochschulen berücksichtigt viel zu wenig die Bedeutung von «Versuch und Irrtum», d. h. des Fehlermachens. Ist der ehemalige Gymnasiast einmal an der Hochschule, so ist er bereits gut geübt im Verbergen von Fehlern. Gerade bei unserem Vorgehen war es jedoch wichtig, daß der Student angstfrei mit Ungewißheit und dem Fehlermachen umgehen kann. Es war daher unsere Aufgabe, die Studenten zu ermutigen und, falls Denk- und Arbeitsstörungen aus den oben genannten Gründen vorlagen, mit ihnen darüber zu sprechen. So wurde es möglich, aus der Diskussion der Fehler zu lernen - ein wichtiger Teil jeder seriösen Forschungsarbeit.

Hinzu kommt, daß sich Praxis nicht einfach in Worten vermitteln läßt, wie man an den folgenden Beispielen sehen kann. Aus der Praxis (d. h. von Gemeinden, Ämtern, Firmen) kommen selten klare Fragen, sondern Probleme, die irgendwie gelöst werden sollen. Oder: Der Praktiker muß in der Lage sein, seine Rolle je nach Erfordernissen zu ändern: wie gelangt er zu Unterlagen, wie beurteilt er Kontaktmöglichkeiten? Denn es spielt eine große Rolle, wie und an wen man welche Frage richtet. Entsprechend fallen die Antworten aus. Und weiter: Die Erfahrung, daß in einer bestimmten Zeitlimite ein Problem gelöst oder daß eine Feldarbeit sorgfältig vorbereitet werden muß, kann den Studenten nicht abgenommen werden.

Ein weiterer Vorteil des Gruppenunterrichts liegt ja, wie bereits in Kap. 2 erwähnt, in der Schulung der Kooperationsfähigkeit. Dazu konnten wir verschiedene Erfahrungen machen. Grundsätzlich war bei den meisten Studenten die Bereitschaft vorhanden, in der Gruppe zu arbeiten. War dies einmal nicht mehr der Fall, so waren interne Konflikte aufgetreten wie Dominanzprobleme, ungenügende Absprache, zu häufiges Fehlen eines Mitglieds sowie mangelnde Bereitschaft, die gruppendynamischen Probleme zu lösen. Dies verhinderte ein ausgewogenes Arbeits- und Bestimmungsverhältnis und beeinträchtigte die Arbeitsfähigkeit der Gruppe. In einer Gruppe war es uns daher nicht gelungen, die Konflikte zwischen den Teilnehmern zu lösen. Als Resultat wurden zwei unterschiedliche Arbeiten abgegeben. In den andern beiden Gruppen gelang die Kooperation, obwohl die Studenten in der Zusammenarbeit allgemein wenig geschult waren. Sie fanden zu einer Zusammenarbeit und Arbeitsteilung, mit der alle zufrieden waren.

Ganz allgemein bestanden auch unterschiedliche Erwartungen an die Gruppe, teils erwarteten die Studenten sehr wenig von einer Zusammenarbeit in der Gruppe, teils hatten sie das Gefühl, es könnten alle Fragen in der Gruppe gelöst werden. Die auftretenden Probleme waren, neben gruppendynamisch bedingten, in erster Linie solche der Arbeitsteilung und der Effizienz.

Die Feldwoche wurde in der ersten Woche des Sommersemesters durchgeführt. Sie wurde als Höhepunkt erlebt und wirkte stark motivierend für die weitere Arbeit. Während dieser Phase der Datenerhebung im Feld war die Betroffenheit und Identifikation mit der Forschungsfrage am größten. Zudem war die Entmutigung im Gegensatz zur Phase der Formulierung von Forschungsfrage und -plan stark zurückgegangen, da die Arbeitsaufträge nun klar formuliert waren oder mit Hilfe der Leiter in dieser Woche noch klarer ausgearbeitet wurden und sogleich im Feld erprobt werden konnten. Zwei Gruppen wurden bei der ersten Feldbegehung von der Dozentin und dem Dozenten begleitet, um so gemeinsam das Vorgehen zu klären und zu zeigen, worauf bei den Erhebungen besonders geachtet werden sollte. Die Gruppe, die von Anfang an relativ rasch eigenverantwortlich forschte, stellte im Laufe der Woche ihre Vorgehensweise und die noch offenen Fragen zur Diskussion. Nach der Arbeit im Felde trafen wir uns jeden Abend zu einem kurzen Protokollreferat und diskutierten das weitere Vorgehen.

Die Feldwoche hatte auch gruppendynamisch positive Auswirkungen, z. B. was die Beziehungen der Studenten untereinander und zu der Dozentin und dem Dozenten betraf, da die Möglichkeit bestand, über die Unterrichtsstunden hinaus sich besser kennenzulernen und allfällige hierarchische Strukturen abzubauen. Ähnliches gilt für die Dozentin und den Dozenten. Es war uns möglich, die Lernchancen zu ergreifen, die das Team-Teaching bietet (DIEMWILle 1986: 177, vergl. Zitat in Kap. 2) und in Diskussionen über fachliche und didaktische Fragen unseren Unterricht zu verbessern. 
Organisatorisch zeigte sich in der ersten Fallstudie, daß die Erhebung im Feld sinnvollerweise in einem zweitägigen Blockkurs am Ende des Wintersemesters erprobt werden sollte. So lassen sich allfällige Schwierigkeiten (fehlende Unterlagen usw.) rechtzeitig erkennen. In der zweiten Fallstudie wurde dieser Blockkurs durchgeführt, was sich sehr bewährte.

Auswertung: Die Feldwoche war ein optimaler Einstieg ins zweite Semester der Fallstudie. Nach der Rückkehr konnte mit der Auswertung oder, wo noch nötig, mit der Beschaffung letzter Daten begonnen werden. Dieses Semester wurde sehr arbeitsintensiv, da es das erste Mal war, daß die Studenten einen größeren Forschungsbericht schreiben mußten. Damit sie sich auf die Datenauswertung und Berichterstattung konzentrieren konnten, erhielten sie eine Wegleitung, welche ihnen den formalen Aufbau eines Forschungsberichts, wie er bereits im Laufe des Seminars in einer Kurzvorlesung zum Thema Arbeitsmethoden vermittelt wurde, zusammenfassend darstellte.

Die Dozentin und der Dozent begleiteten diese Phase mit fachkritischen Hinweisen. Wo es nötig war, wurden weitere Fachreferenten beigezogen. Nach der Abgabe eines Rohberichtes, der kritisch beurteilt und mit den Studentinnen und Studenten besprochen wurde, erstellten sie eine ausformulierte Fassung. Diese wurde wiederum inhaltlich und formal korrigiert und diente als Vorlage für den Bericht.

Präsentation: Die Ergebnisse der Untersuchungen wurden am Amt für Umweltschutz und Wasserwirtschaft des Kts. Thurgau sowie am Geographischen Institut der ETH im Rahmen eines Kolloquiums vorgestellt. Es war für alle Teilnehmer ein sehr befriedigender Abschluß. Am Geographischen Institut stellten sich die Studenten kritischen Fragen. Auch die Vertreter des obigen Amtes, welche bei der Durchführung der Fallstudie mehrfach und in verdankenswerter Weise Unterstützung gewährten, drückten ihre Überraschung darüber aus, wieviel in der relativ kurzen Zeit erreicht worden war. Der schriftliche Bericht erschien ein halbes Jahr später (BUFF KELLER und PFISTER, Hrsg. 1988). Er wurde wie folgt beurteilt: «... Die Arbeiten (sind) von hoher Qualität und großem Nutzen ... Wir werden die Erkenntnisse auch im Bundesamt verwerten» (LÄssKER 1988).

\section{Schlußfolgerungen}

Insgesamt kann die erste Fallstudie in Geographie, die am Geographischen Institut der ETHZ durchgeführt wurde, als in jeder Hinsicht erfolgreich bezeichnet werden.
Dozenten: Wir können nach dieser intensiven $\mathrm{Zu}$ sammenarbeit sagen, daß wir sehr viel voneinander lernen konnten und $d a ß$ durch die gemeinsame Lehrtätigkeit ein gegenseitiges Vertrauensverhältnis entstand. Da wir uns von Anfang an in den grundlegenden Fragen betreffend Team-Teaching, wissenschaftstheoretischem Hintergrund, Interdisziplinarität usw. einig waren und mit viel Engagement an unsere neue Aufgabe herangingen, war es uns auch möglich, mit der Zeit auftretende Schwierigkeiten, Vorbehalte und Unsicherheiten auszudrücken und nach Lösungen zu suchen. Dies wirkte sich für die zweite Fallstudie sehr fruchtbar aus, da wir ein gut eingespieltes Team geworden waren, in dem viele der in Kap. 2 genannten Unsicherheiten abgebaut werden konnten.

Wir beschlossen, noch früher mit den Gruppenarbeiten anzufangen, damit möglichst schnell ein identifikationsorientiertes Lernen stattfinden kann. Sind einmal die fachlichen Probleme (Themenwahl, Methoden usw.) gelöst und ist ein gutes Team-Teaching zustandegekommen, stehen als Hauptprobleme die Gruppendynamik, das soziale Lernen und das Eingehen auf die individuelle Lernsituation der Studentinnen und Studenten im Vordergrund.

Gruppenarbeit: Wie BÜNTE-LUDWIG und CZERWENKA (1984: 19) bei ihren Seminarien mit Studenten feststellten, war die Gruppendynamik selten ein Thema der Arbeitsgruppen. Diese Beobachtung konnten auch wir machen. Es ist deshalb eine weitere Zielsetzung für zukünftige Fallstudien, den Studenten zu einem bewußteren Wahrnehmen und Formulieren der gruppeninternen Interaktionen zu verhelfen. Gerade hier kann der Dozent bei entsprechender Ausbildung in Einzelfällen entscheidend Hilfe für das weitere Studium und Lernen anbieten. Zum Beispiel war eine Gruppe während längerer Zeit nicht in der Lage, unsere sachliche Kritik und Hilfestellung zu akzeptieren. Das Resultat war ein knapp genügender Schlußbericht. Nach einer ausführlichen und offenen Diskussion ihres Berichts war es einigen Studenten dieser Gruppe möglich, ihre z. T. falsche Einstellung zum Lernen zu erkennen, über ihren eigenen Schatten zu springen und aus den gemachten Fehlern die Konsequenzen zu ziehen: Sie führten eine neue, sehr gute Untersuchung durch. Den Wunsch nach immer mehr Selbstbestimmung der Studenten (BÜNTE-LUDWIG und CZERWENKA 1984: 19) konnten wir in unterschiedlichem Maße beobachten. Obwohl faktisch die Verantwortung bei uns lag, gab es Studenten, welche die Initiative ergriffen und eigenverantwortlich handelten. Es ist daher empfehlenswert, wenn mindestens einer der Dozenten eine gründliche didaktische Ausbildung absolviert hat. Ebenso wichtig erscheint uns die Persönlichkeitsschulung der Dozenten, sind sie doch gerade bei den genannten Unterrichtsformen sehr 
oft mit ungewissen Situationen konfrontiert und müssen unbewußte Ängste abbauen, wenn sie in einer solchen Situation bestehen wollen. Wie wir jedoch erfahren konnten, haben wir beide sowohl über unsere Erfahrungen im Team-Teaching als auch in der nicht immer einfachen Auseinandersetzung mit den Studenten viel gelernt.

Die meisten Studenten erlebten das prozeßorientierte Lernen in der Gruppe positiv und waren mit ihren als Bericht vorliegenden Resultaten sehr zufrieden. Sie haben sowohl in fachlicher als auch in sozialer und politischer Hinsicht viele Erfahrungen gemacht, die ihnen nach dem Studienabschluß den Einstieg ins Berufsleben sicher erleichtern werden.

Wir danken der Abteilung für Naturwissenschaften und dem Geographischen Institut der ETHZ, daß wir im neuen Geographiestudium das Fach «Geographische Fallstudie» aufbauen und durchführen konnten. Im weiteren danken wir den GeographieStudenten des 5./6. Semesters 1986/87 für ihre engagierte Teilnahme und $\mathrm{H}$. Lang (Abt. Hydrologie des Geographischen Institutes ETH) für seine fachliche Unterstützung während der Projektzeit. M. Steinmann (Abb. 1), M. Werfeli und R. Heer (Abb. 2) danken wir für das Zeichnen der Abbildungen.

\section{Literatur}

BUFF KELLER E. (1987): Das Berggebiet. Abwanderung, Tourismus, Regionale Disparitäten. Geographica Bernensia S12, Unterrichtseinheit für die Sekundarstufe II. Geographisches Institut der Universität Bern, Bern.

BUFF KELLER E. und PFISTER P. (Hrsg.) (1988): Geographische Fallstudie 1986/87. Berichte und Skripten Nr.33, Geographisches Institut der ETH, Zürich.

BÜNTE-LUDWIG CH. und CZERWENKA K. (1984): Das gruppendynamische Seminar. Lüneburger Hochschulzeitung Nr. 4, S. 16-19. (Vgl. auch Czerwenka, K., 1982: Wirklichkeitserfahrung in der Schule, Donauwörth.)

DIEM-WILLE G. (1986): Gruppenunterricht. In: Husa K., Vielhaber Ch. und Wohlschlägl H., Hrsg.: Beiträge zur Didaktik der Geographie. Festschrift Ernest Troger zum 60. Geburtstag, Band 2. F. Hirt, Wien.

LEMERHOFER M. (1986): Gruppenunterricht und soziales Lernen in "Geographie und Wirtschaftskunde". GW Unterricht Nr. 23, Wien.

LÄSSKER, A. J. (1988): Brief des Direktors des Bundesamts für Wasserwirtschaft (vormals Chef des Amtes für Umweltschutz und Wasserwirtschaft des Kts. Thurgau) an das Geographische Institut der ETH Zürich. 